\title{
Estimation of shear wave anisotropy of transversely isotropic medium by full waveform inversion
}

\author{
Satoshi FUSE ${ }^{1}$, Hitoshi MIKADA ${ }^{1}$ and Junichi TAKEKAWA ${ }^{1}$ \\ ${ }^{1}$ Dept. of Civil and Earth Res. Eng., Kyoto University
}

\begin{abstract}
It is necessary to obtain an accurate underground velocity structure to grasp the image of subsurface in seismic survey. Among various estimation methodologies, borehole logging is one of the best ways accurately to estimate the rock elastic properties of the ground around the wellbore. In the conventional study, the combination of Alford rotation with slowness time coherence (STC) has been applied to estimate both the formation velocity and the azimuth angles under the existence of azimuthal anisotropy in the formation. However, it has been revealed the approach with Alford rotation could fail or gives improper estimates when the axis of symmetry of the anisotropic later does not lie in the plane orthogonal to the well axis.

In this study, we conduct numerical simulation for transversely isotropic medium (TI) which has 5 independent stiffness elements in 3-dimensional logging model. In recent years, full waveform inversion (FWI) has been focused which could estimate physical properties by using all information of waveforms. We investigate the feasibility of FWI to detect the orientation and dip of TI. We introduce the Euler angles into TI to estimate the stiffness parameters by FWI instead of estimating the stiffness parameters as orthorhombic medium under a hypothesis that the stable solution can be obtained by introducing the Euler angles. This approach can reduce unknowns in FWI, i.e. computational efficiency and stability of inversion could be improved. The result clearly indicates that the FWI for anisotropic medium is effective in order to detect the shear wave anisotropy and stable solution could be obtained according to misfit function even when the anisotropic layer has the dip and orientation.
\end{abstract}

\section{INTRODUCTION}

Seismic anisotropy exists underground due to the opening and closing of fractures and the selected crystal structure. It is difficult to obtain the velocity structure in complicated sedimentary structures and formations with seismic anisotropy. Phase velocity varies due to the propagation direction in anisotropic formation. Therefore, it is important to estimate the anisotropic properties of formation.

The number of anisotropic parameters in stiffness matrix is five in transversely isotropic medium. The elements of the matrix change complicatedly due to the change in the anisotropic orientation and dip (Figure 1). To estimate the degree of the anisotropy it is necessary to adopt the whole 21 elements as parameters which cause instability of inverse analysis. Therefore we hypothesize that an optimal solution can be stably obtained by introducing Euler angles. This approach can reduce the unknowns in FWI that results in improving computational efficiency and stability of inversion.

Full Waveform Inversion has recently been adopted to estimate physical properties of the formation by using all information of waveforms such as amplitude and phase ${ }^{1)}$. The previous research $^{2)}$ confirmed that the received waveform changes due to the azimuth of anisotropy. By applying FWI to this waveform, seismic anisotropy could be estimated. In this research, numerical experiment is conducted in a $3 \mathrm{D}$ sonic logging model with the aim of estimating elastic elements, orientation and inclination.

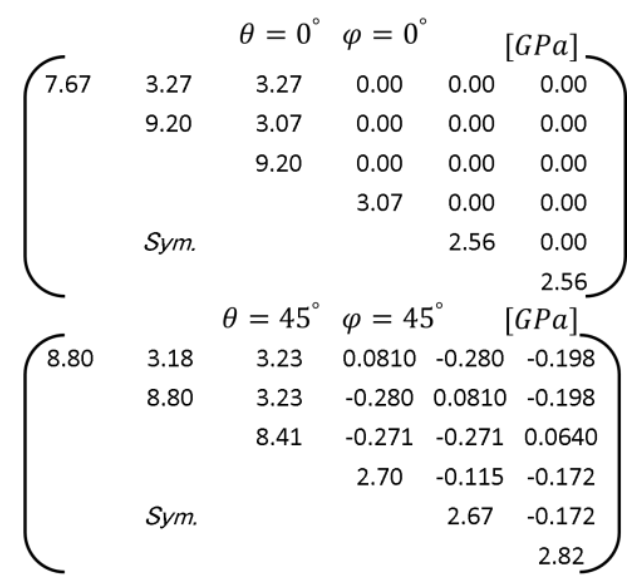

Figure 1 The complexity of Stiffness Matrix of azimuthal anisotropy 


\section{METHOD}

\section{(1) Governing Equation}

To express seismic wave propagation, we use the Hamiltonian particle method with staggered particles $(\mathrm{HPM})^{3)}$. HPM is one of the particle methods which has high computational efficiency and can be applied to flexible models. The governing equation is described as follows.

$$
\rho_{i} \Delta B_{i} \frac{\partial \boldsymbol{v}_{\boldsymbol{i}}}{\partial t}=-\frac{\partial H}{\partial \boldsymbol{x}_{\boldsymbol{i}}}
$$

where $\rho_{i}, \Delta B_{i}, \boldsymbol{v}_{\boldsymbol{i}}$ is the density, volume and particle velocity of $i$ th particle and $H$ is determined by the sum of kinetic and strain energy.

The stress-strain relationship is related by the stiffness matrix. Although the number of the independent elements of the stiffness matrix is 2 in isotropic medium, the stiffness matrix of TI is represented by the 5 independent elements.

$$
\boldsymbol{C}=\left(\begin{array}{cccccc}
C_{11} & C_{13} & & C_{13} & & \\
& C_{33} & C_{33}-2 C_{44} & 0 & \\
& & C_{33} & & \\
& & & C_{44} & \\
& & & & \\
& & & & C_{66} & \\
& & & & & C_{66}
\end{array}\right)
$$

\section{(2) Numerical Model}

As a three-dimensional acoustic logging model, we assumed a model which contains a set of source and receiver in a uniform anisotropic layer. As for source, a pair of orthogonal dipole sources is set and records the waveform using a three component receiver. As the physical properties of the anisotropic layer, we assumed a formation with $15 \%$ seismic anisotropy for both $\mathrm{P}$ and $\mathrm{S}$ wave (Figure 2).

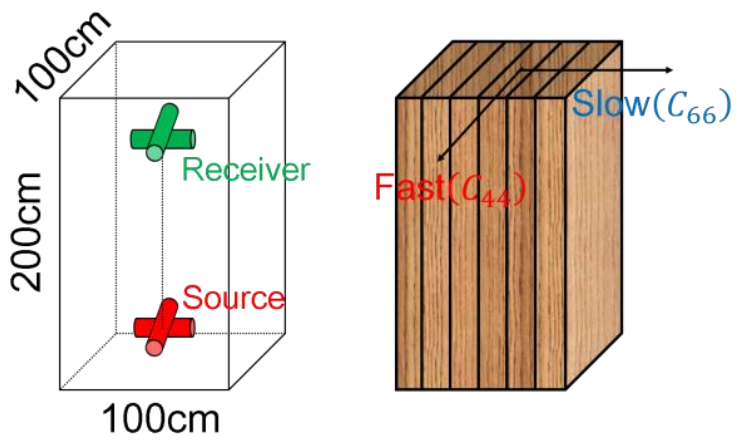

Figure 2 Numerical Model
Table 1 Formation property

\begin{tabular}{r|l}
\hline \multicolumn{1}{l|}{ Formation } & \\
\hline P wave velocity $[\mathrm{m} / \mathrm{s}]$ & \\
Isotropy & 2000 \\
Anisotropy & $1690(15.5 \%)$ \\
S wave velocity [ $\mathrm{m} / \mathrm{s}]$ & \\
Isotropy & 1154 \\
Anisotropy & $975(15.5 \%)$ \\
Density [g/cm $\left.{ }^{3}\right]$ & 2.30 \\
\hline Elastic modulus & \\
\hline$C_{11}$ [GPa] & 9.20 \\
$C_{13}$ [GPa] & 3.27 \\
$C_{33}$ [GPa] & 7.67 \\
$C_{44}$ [GPa] & 2.56 \\
$C_{66}$ [GPa] & 3.07 \\
\hline
\end{tabular}

\section{(3) Full Waveform Inversion}

FWI is a method to estimate a true model with high accuracy by updating model parameters so as to minimize the misfit between observed and calculated waveform. The misfit function is defined by the following equation.

$$
E=\frac{1}{2} \sum\left(d_{o b s}-d_{c a l}\right)
$$

The model parameter is updated by the following.

$$
\boldsymbol{m}_{k+1}=\boldsymbol{m}_{k}+a_{k} \delta \boldsymbol{m}_{k}
$$

For the kth iteration, the model parameters, step length, and gradient are defined as $\boldsymbol{m}_{k}, a_{k}$ and $\delta \boldsymbol{m}_{k}$, respectively. The model was updated using the conjugate gradient method.

For efficient estimation of the elastic element, the gradient of the elastic element of the misfit function $E$ is theoretically obtained based on the wave equation $^{4}$.

$$
\frac{\partial E}{\partial C_{i j k l}}=\int \frac{\partial u_{i}}{\partial x_{j}} \frac{\partial \psi_{k}}{\partial x_{l}} d t
$$

where $C_{i j k l}$ is the elastic element, $u_{i}$ is the wave field propagated from the source, and $\psi_{k}$ is the wave field from the receiver. 


\section{RESULTS}

\section{(1) FWI for Shear wave anisotropy}

FWI is performed to estimate the shear wave anisotropy. The anisotropy of the shear wave is represented by the elements $C_{44}$ and $C_{66}$ of the elastic matrix. The true model is estimated from the received waveform using a pair of orthogonal dipole sources and a 3-component receiver. The initial anisotropic model is $10 \%$ disturbed in terms of stiffness elements.

As a result, the stiffness elements related to fast and slow shear can be estimated accurately within about 5 iterations.

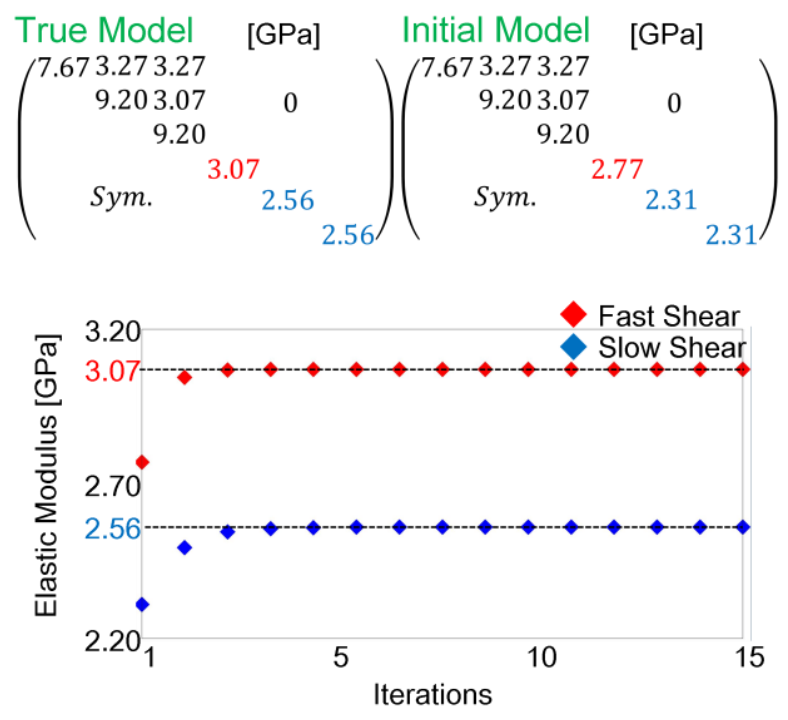

Figure 3 FWI for Shear Wave Anisotropy

\section{(2) Misfit function}

Visualizing misfit function in 3-dimensional block is an efficient way to evaluate the stability of FWI for multi parameterization. We set the true anisotropic model as $C_{44}$ is $3.07 \mathrm{GPa}$, orientation is 45 degrees and dip is 45 degrees. We evaluate the misfit function by changing $C_{44}$ from 2.70 to 3.40 $\mathrm{GPa}$, orientation from 0 to 90 degrees and dip from 0 to 90 degrees. The 3 -dimensional block is cut in the arbitrary plane (Figure 4,5,6,7). By using the iterative process, the true value could be obtained.

Table 2 True Model

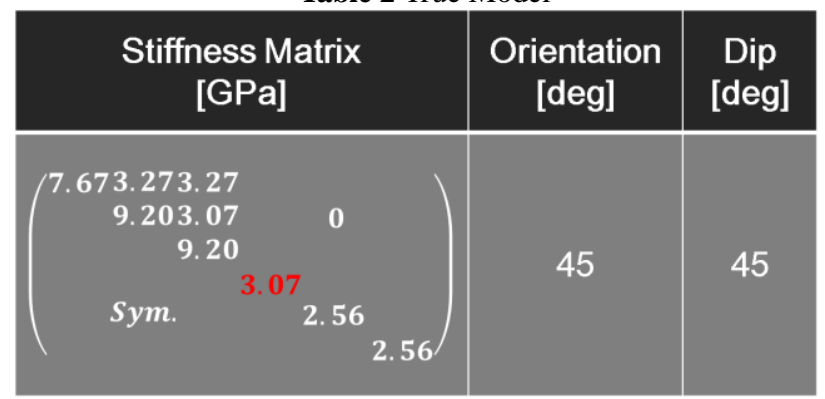

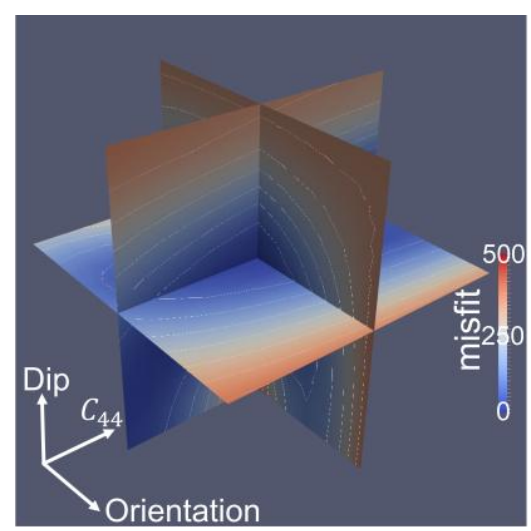

Figure 4 3D block of Misfit

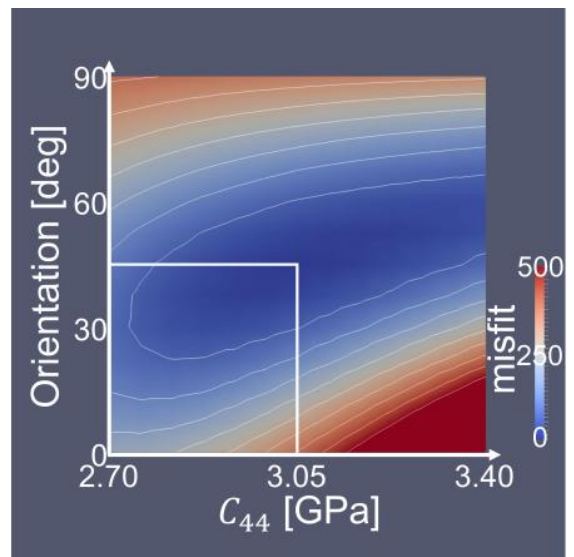

Figure 5 Misfit plane of $C_{44}$ and orientation

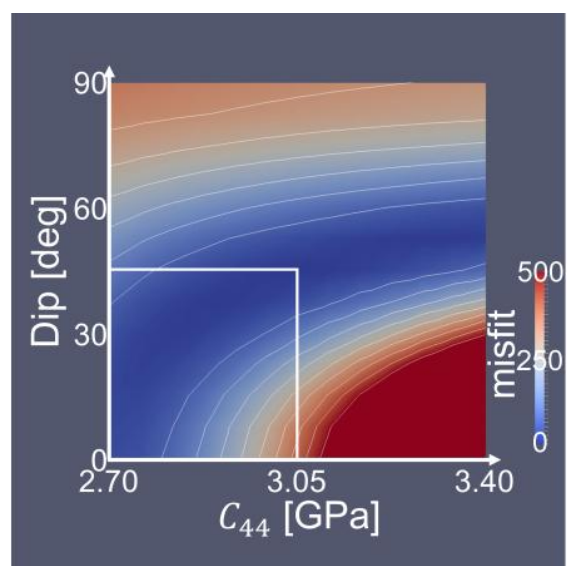

Figure 6 Misfit plane of $C_{44}$ and dip

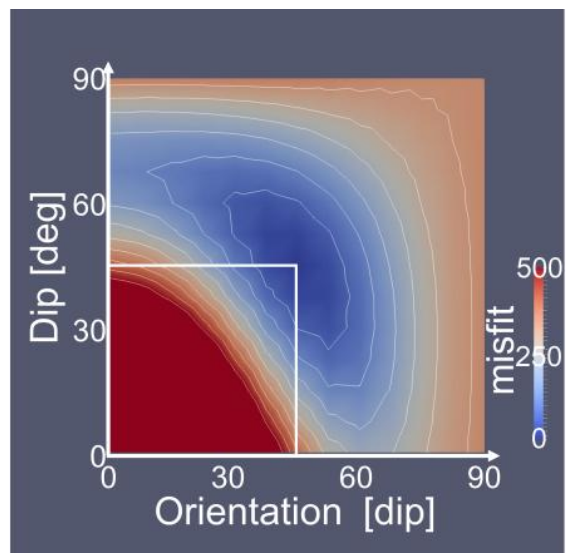

Figure 7 Misfit plane of orientation and dip 


\section{CONCLUSION}

Since the stiffness matrix becomes complex due to the orientation and dip of anisotropic layer, direct estimation of the 21 stiffness elements should be unstable. Then, we suggest the technique that is introducing the orientation and dip parameters with 5 independent stiffness elements to solve the instability and improve the computational efficiency. FWI is adopted to the data obtained in the anisotropic medium by 3D numerical simulation.

The inversion result clearly indicates the shear wave anisotropy can be estimated with high accuracy by using two orthogonal dipole sources. The misfit for $C_{44}$, orientation and dip are visualized as 3D block. The misfit decreases as it approaches the true value as one global minimum. This result suggests the possibility of obtaining true parameters of anisotropic layer.

FWI has some advantages; i) we can estimate properties with only a pair of source and receiver, ii) the properties of multiple layers could be estimated, iii) it could be applied to deviated wells.

\section{REFERENCES}

1) Tarantola, A., 1986, A strategy for nonlinear elastic inversion of seismic reflection data,Geophysics, 51 (10), 1893-1903.

2) Fuse, S., Mikada, H., Takekawa, J., 2016, Full waveform inversion of cross-dipole acoustic waveforms, International Petroleum Tecnology Conference 2016, IPTC-18726-MS.

3) Takekawa, J., H. Mikada, and T. Goto, 2014, A Hamiltonian particle method with a staggered particle technique for simulating seismic wave propagation, Pure and Applied Geophysics, 171 (8), 1747-1757.

4) Kamath, N., \& Tsvankin, I., 2016, Elastic full-waveform inversion for VTI media: Methodology and sensitivity analysis, Geophysics, 81 (2), C53-C68

5) Alford, R. M. 1986, Shear data in the presence of azimuthal anisotropy: Dilley, Texas, 56th Annual Meeting, Society of Exploration Geophysicists, Expanded Abstract, 476-479

6) Bergslid, T. S., Birger Raknes, E., and Arntsen, B., 2015, The influence of anisotropy on elastic full-waveform inversion. 85th Annual Meeting, Society of Exploration Geophysicists, Expanded Abstract, 1425-1429

7) Butzer, S., Kurzmann, A., Bohlen, T., 2013, 3D elastic full-waveform inversion of small-scale heterogeneities in transmission geometry, Geophysical Prospecting, 61 (6), 1238-1251

8) Cerjan, C., Kosloff, D., Kosloff, R., and Reshef, M., 1985, A nonreflecting boundary condition for discrete acoustic and elastic wave equations, Geophysics, 50 (4), 705-708.

9) Ishikura, K., H. Mikada, T. Goto and J. Takekawa, 2014, Estimation of fluid contact in terms of attenuation, 84th Annual Meeting, Society of Exploration Geophysicists, Expanded Abstracts, 623-627.

10) Kimball, C. V., and Marzetta, T. L., 1984, Semblance processing of borehole acoustic array data, Geophysics, 49 (3), 274-281
11) Kitsunezaki, C., 1981, A new method for shear-wave logging, Geophysics, 45 (5), 1489-1506.

12) Saenjer, E. H., and Bohlen, T., 2004, Finite-difference modeling of viscoelastic and anisotropic wave propagation using the rotated staggered grid, Geophysics, 69 (8), 583-591.

13) Thomsen, L., 1986, Weak elastic anisotropy, Geophysics, 51 (10), 1954-1966. 